\title{
Prevalência de obesidade em escolares de 7 a 12 anos de uma cidade Serrana do RS, Brasil
}

\author{
Prevalence of obesity and overweight in schoolchildren aged 7 \\ to 12 years from a city in southern Brazil
}

\author{
Ricardo Rodrigo Rech ${ }^{1}$ \\ Ricardo Halpern ${ }^{2,3}$ \\ Cristine Boone Costanzi 2 \\ Mauren Lúcia de Araújo Bergmann 2 \\ Lidiane Requia Alli 2 \\ Airton Pozo de Mattos 2 \\ Leandro Trentin \\ Lisiane Reis Brum ${ }^{1}$
}

1 Universidade de Caxias do Sul. Caxias do Sul, RS. Brasil.

2 Universidade Luterana do Brasil. Canoas, RS. Brasil

3 Universidade Federal Ciências da Saúde. Porto Alegre, RS. Brasil

Recebido em 03/11/08 Revisado em 13/02/09 Aprovado em 18/09/09
Resumo - A obesidade e o sobrepeso vêm aumentando suas prevalências em todo o mundo nos últimos anos. O objetivo do presente estudo foi estimar a prevalência de obesidade e sobrepeso em escolares de 7 a 12 anos (meninos e meninas), de uma cidade serrana do RS e verificar as possíveis associações com as seguintes variáveis: classe socioeconômica, aptidão aeróbica, hábitos alimentares e hábitos de lazer (sedentários e atividade física). Foram avaliados 1.442 escolares, através de um estudo transversal. As variáveis antropométricas estudadas foram massa corporal total e estatura. A obesidade foi definida pelo IMC, segundo sexo e idade. Foi realizada uma análise descritiva, bivariada e multivariada entre as variáveis independentes e o desfecho. As prevalências de obesidade e sobrepeso foram de $8 \%(\mathrm{IC}=7,54-8,45)$ e 19,9\% ( $\mathrm{IC}=19,62-20,17)$ respectivamente. As variáveis aptidão aeróbica $(\mathrm{p}<0,000-\mathrm{RP}=0,59)$, prática de esportes fora do horário escolar $(\mathrm{p}=0,005$ - $\mathrm{RP}=1,41$ ), hábitos sedentários (televisão, vídeo game e computador) ( $\mathrm{p}=0,004-\mathrm{RP}=1,50$ ) e nível socioeconômico alto $(\mathrm{p}<0,000-\mathrm{RP}=2,64)$ apresentaram associação estatística significante com obesidade e sobrepeso mesmo após ajuste na análise multivariada. As variáveis referentes a hábitos alimentares foram significativas na análise bivariada, mas não se mantiveram no modelo multivariado. As prevalências de obesidade e sobrepeso da população estudada encontram-se elevadas e devem ser motivo de preocupação das autoridades de saúde do município.

Palavras-chave: Obesidade; Sobrepeso; Criança; Fatores de risco.

Abstract - The prevalence of obesity and overweight has been increasing worldwide over the last few years. The purpose of this study was to estimate the prevalence of obesity and overweight in schoolchildren aged 7 to 12 years from a mid-sized city in southern Brazil, and to determine possible associations with socioeconomic status, aerobic fitness, dietary habits, and leisure activities (sedentary and physical activity). A cross-sectional study was conducted and 1,442 children were evaluated regarding body weight and height. Obesity was defined based on BMI for gender and age. Descriptive, bivariate and multivariate analyses were carried out between the independent variables and outcome. The prevalence of obesity and overweight was $8 \%$ (CI: 7.54-8.45) and 19.9\% (CI: 19.62-20.17), respectively. Aerobic fitness ( $p<0.000, P R=0.59)$, practicing out-of-school time sports ( $p=0.005, P R=1.41)$, sedentary habits ( $T V$, video games and computer) $(p=0.004$, $P R=1.50)$, and high socioeconomic level $(p<0.000, P R=2.64)$ were significantly associated with obesity and overweight in the adjusted multivariate model. Dietary habits were directly associated with obesity and overweight, but did not remain in the final multivariate model. The prevalence of obesity and overweight in this population was high and should be a matter of concern to city health authorities.

Key words: Obesity; Overweight; Schoolchildren; Risk factors. 


\section{INTRODUÇÃO}

O aumento da prevalência de obesidade e sobrepeso vem ocorrendo em todas as faixas etárias, nos países desenvolvidos e em desenvolvimento. Nos últimos anos, no Brasil, houve um aumento da prevalência de excesso de peso e a diminuição da prevalência de desnutrição, caracterizando a transição nutricional' ${ }^{1}$.

O excesso de peso na infância pode ocasionar complicações ortopédicas como desconfortos musculoesqueléticos, fraturas e dificuldades de mobilidade ${ }^{2}$. Além disso, a obesidade também pode acarretar complicações metabólicas, psicossociais e respiratórias ${ }^{3}$, diminuição da qualidade de vida e doenças cardiovasculares ${ }^{4}$.

Estudos recentes têm mostrado que o sobrepeso e a obesidade comprometem a saúde desde a infância, estendendo-se pela adolescência e idade adulta. Deshmukh-Taskar et al. ${ }^{5}$ destacam que $61,9 \%$ das crianças, por eles avaliadas e que foram classificadas como obesas, continuaram obesas na fase adulta da vida. Freedman et al..$^{6}$ encontraram alta associação entre obesidade na infância e obesidade e adiposidade na vida adulta.

Os prováveis fatores para o aumento da obesidade infantil são a diminuição no nível de atividade física e a qualidade da dieta. O número de horas assistindo televisão, número de refeições, o hábito de tomar café da manhã ou não, também são hábitos que estão associados à obesidade infantil?

As diferenças geográficas, econômicas e culturais expressam taxas diversas em relação à obesidade. Esta diversidade aponta a necessidade de obtenção de informações sobre obesidade infantil nas diferentes cidades e regiões do país ${ }^{8}$. O presente estudo teve por objetivo estimar a prevalência de obesidade e sobrepeso em escolares de 7 a 12 anos de idade (meninos e meninas) em uma cidade serrana do RS, e verificar as possíveis associações com classe socioeconômica, aptidão aeróbica relacionada à saúde, hábitos alimentares e hábitos de lazer (hábitos sedentários e de atividades físicas).

\section{PROCEDIMENTOS METODOLÓGICOS}

Trata-se de um estudo transversal do tipo inquérito epidemiológico de base escolar. A população alvo foram os escolares de 07 a 12 anos matriculados no turno diurno das redes de ensino pública e privada, de uma cidade serrana do RS. A população de escolares de 07 a 12 anos de idade, no ano de 2005, de acordo com dados da Secretaria Estadual de Edu- cação, foi de 33.241 escolares (população elegível para o estudo). Com base em estudos análogos, foi estimada para o cálculo de tamanho da amostra uma prevalência média de obesidade + sobrepeso de $20 \% \%^{1,7,-13}$. Com um intervalo de confiança de $95 \%$, um poder de $80 \%$ e um erro aceitável de 3\%, seriam necessárias 669 crianças. Antecipando-se a possíveis perdas e recusas e utilizando um efeito de delineamento 2, duplicou-se o tamanho da amostra. No total, foram sorteadas 1573 crianças. O critério de amostragem adotado foi por conglomerados; cada escola foi considerada um conglomerado e todas as crianças da escola que se encontravam dentro da faixa etária de 07 a 12 anos receberam o termo de consentimento. Todas as escolas participaram do sorteio e tiveram as mesmas chances de participar do estudo de acordo com o número de alunos matriculados na faixa etária de 07 a 12 anos. Foram adotados os seguintes critérios de exclusão: crianças apresentando qualquer deficiência física, asma, idade superior a 12 anos e inferior a 7 anos.

Foi utilizado um questionário estruturado (entrevista) para a avaliação das variáveis: classe socioeconômica, sexo, idade (em anos), hábitos alimentares e hábitos de lazer (hábitos sedentários e de atividade física). Os dados foram coletados diretamente através de entrevista com os alunos.

O questionário referente aos hábitos alimentares apurou questões sobre a alimentação realizada no dia anterior, desde o café da manhã até a última refeição da noite. Após os questionamentos, foi realizado o somatório de quantas refeições a criança realizava diariamente (café da manhã, lanche da manhã, almoço, lanche da tarde, jantar e ceia), assim como o hábito de tomar ou não café da manhã. As questões referentes a tomar café da manhã ou não tomar e o número de refeições diárias foram fatores que estiveram associados com o excesso de peso em estudos previamente realizados ${ }^{7,14}$.

A variável dos hábitos de lazer apurou duas questões: 1) os hábitos sedentários de assistir televisão, jogar video-game ou permanecer em frente ao computador (somatório das atividades em horas diárias); e 2) a prática de esportes fora do horário escolar (questão objetiva com resposta possível sim ou não).

As questões referentes aos hábitos alimentares e esportes fora do horário escolar foram criadas pelos autores e testadas no projeto piloto.

Informações referentes à classe socioeconômica foram estratificadas, utilizando-se a proposta de Barros e Victora ${ }^{15}$ que considera a utilização de 13 variáveis para produzir o Indicador Econômico 
Nacional (IEN). O IEN foi baseado no censo demográfico de 2000.

Para a avaliação da aptidão aeróbica, foi realizado o teste de corrida/caminhada de 9 minutos. $\mathrm{O}$ teste consiste em percorrer a maior distância possível no tempo de 9 minutos. É um teste de corrida, mas as crianças podem caminhar durante o teste se assim desejarem. Os escolares foram classificados como estando abaixo, acima ou na zona de aptidão aeróbica relacionada à saúde de acordo com a distância em metros percorrida no teste. Todas as instruções para o teste encontram-se no manual do Projeto Esporte Brasil ${ }^{16}$.

Foram medidos nos escolares a massa corporal total e a estatura. Para a medida de massa corpórea, foi utilizada balança portátil digital da marca Plena, com precisão de $100 \mathrm{~g}$. Para a medida da estatura, foi utilizado estadiômetro fixado na parede e esquadro. O índice de massa corporal (IMC) foi obtido pela divisão da massa corporal total pela estatura elevada ao quadrado $\left(\mathrm{IMC}=\right.$ massa corporal total/estatura $\left.{ }^{2}\right)$. A obesidade e o sobrepeso foram definidos através dos pontos de corte de IMC para sexo e idade desenvolvidos pelo International Obesity Task Force - IOTF. Os pontos de corte para meninos e meninas e suas respectivas idades se encontram no artigo de Cole et al. ${ }^{17}$.

Para controle de qualidade, foram refeitas $5 \%$ das avaliações (via telefone) de forma aleatória, bem como a dupla digitação dos dados. As variáveis revisadas via telefone foram o nome completo, a idade, a escolaridade do chefe da família e o hábito de tomar ou não café da manhã. Foram sorteados, aleatoriamente, 36 meninos e 37 meninas sem a preocupação da idade dos mesmos. Nenhuma das 73 avaliações via telefone apresentou dados divergentes dos anteriormente coletados. Os dados foram armazenados em um banco de dados formatado em EPIDATA.

Após a verificação da consistência dos dados, o banco foi exportado para o programa SPSS 10 onde foi analisado. Para a análise, os estudantes obesos foram agrupados aos que apresentavam sobrepeso, gerando a variável excesso de peso. Inicialmente, foi realizada uma análise descritiva e após, uma análise bivariada entre as variáveis independentes e o desfecho dicotômico. As variáveis independentes que apresentavam associação significativa com o desfecho até o nível de significância de 5\%, ou aquelas que tinham grande plausibilidade de associação foram incluídas na análise multivariada. Foi realizado teste de colinearidade entre as variáveis que representavam hábitos alimentares e entre as que representavam atividades físicas e foram excluídos da modelagem aquelas que apresentaram essa condição.
Foi realizada uma análise multivariada (regressão logística), controlando os possíveis fatores de confusão em relação ao desfecho de acordo com os níveis hierárquicos definidos no modelo (figura 1). As variáveis independentes foram colocadas em blocos, no modelo, sendo que cada bloco corresponde a um nível hierárquico. Aquelas que apresentavam significância estatística até 20\% foram mantidas no modelo, sendo acrescentadas as variáveis do nível seguinte e assim por diante. Todas as variáveis do modelo teórico foram controladas na análise multivariada como possíveis fatores de confusão.

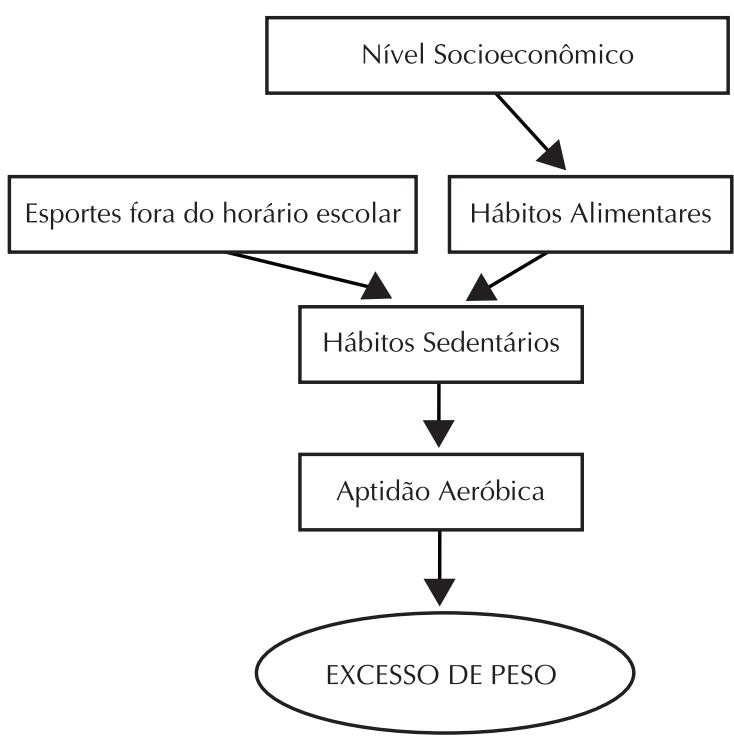

Figura 1 - Modelo teórico hierarquizado adotado para o presente estudo

Quanto à questão ética, todas as crianças que fizeram parte da amostra assinaram o termo de consentimento livre e esclarecido, assim como seus pais ou responsáveis. O estudo foi aprovado pelo Comitê de Ética em Pesquisa da Universidade Luterana do Brasil com número de protocolo 2006-365H.

\section{RESULTADOS}

Das 1573 crianças selecionadas para o estudo, 1442 foram avaliadas. Trinta crianças se recusaram a participar do estudo e 38 não apresentaram o termo de consentimento livre e esclarecido assinado pelos responsáveis. Foram excluídos 45 questionários que apresentaram dados em branco. Também foram excluídas da análise 4 crianças que apresentavam idade inferior a 7 anos e 14 que apresentavam idade superior a 12 anos. Durante o período de avaliação, nenhuma criança relatou ser portadora de deficiência física ou asma. As perdas e recusas somaram 8,4\% ( $\mathrm{n}=131)$. A distribuição da amostra 
foi normal. A amostra ficou distribuída igualmente por sexo, ou seja, 721 meninos (50\%) e 721 meninas. A igualdade nos números para meninos e meninas deu-se por coincidência após o fechamento do banco de dados.

As prevalências de obesidade e sobrepeso foram de $8 \%(\mathrm{IC}=7,54-8,45)$ e $19,9 \%(\mathrm{IC}=19,62$ - 20,17) respectivamente. Não foi encontrada diferença estatística significante para obesidade $\mathrm{e}$ sobrepeso entre meninos e meninas $(\mathrm{p}=0,594-\mathrm{IC}=$ $0,81-1,28)$ e entre as diferentes faixas etárias das crianças avaliadas $(\mathrm{p}=0,863-\mathrm{IC}=0,73-1,12)$. Também não houve diferença entre as redes de ensino pública e privada $(\mathrm{p}=0,325-\mathrm{IC}=0,70-1,30)$.

A tabela 1 apresenta a distribuição da amostra e as prevalências de obesidade e sobrepeso. As características antropométricas da amostra e o teste de aptidão aeróbica, segundo sexo e idade, estão descritos na tabela 2 .

Para a análise bivariada e multivariada, os escolares obesos foram agrupados aos que apresentavam sobrepeso, totalizando $27,9 \%$ de crianças com excesso de peso. A tabela 3 sumariza os resultados da análise bivariada e multivariada, após o ajuste, para possíveis fatores de confusão.

Após a análise multivariada, a prática esportiva fora do horário escolar apresentou associação estatisticamente significativa $(\mathrm{p}=0,005)$ com excesso de peso. Os escolares que não relataram praticar esportes no lazer apresentaram $41 \%$ a mais de chances para excesso de peso $(\mathrm{RP}=1,41-\mathrm{IC}=1,11-1,81)$ em relação àqueles que praticavam.

As crianças que relataram manter hábitos sedentários como permanecer em frente ao computador, assistir televisão ou jogar vídeo game por mais de três horas diárias apresentaram 50\% a mais de chances $(p=0,004)$ para excesso de peso $(\mathrm{RP}=1,50-\mathrm{IC}=1,13-2,00)$.

A aptidão aeróbica mensurada pelo teste de corrida apresentou associação inversa com obesidade e sobrepeso $(\mathrm{p}=0,000)$. Atingir ou ultrapassar a distância estipulada para ficar dentro da faixa saudável da aptidão aeróbica apresentou fator de proteção para obesidade e sobrepeso $(\mathrm{RP}=0,59-\mathrm{IC}=0,46$ $0,77)$. As crianças que atingiram ou ultrapassaram essa faixa apresentaram $41 \%$ menos chances para excesso de peso que as demais crianças.

Em relação à situação socioeconômica, as crianças foram agrupadas de acordo com o indicador econômico nacional (IEN). Cinquenta e nove por cento (691) das crianças estudadas encontramse na classe econômica mais alta, 30,3\% (356) na classe intermediária e 10,7\% (125) na classe mais baixa. A classe socioeconômica apresentou associação direta com excesso de peso e a prevalência de obesidade e sobrepeso aumentou de forma estatisticamente significativa $(\mathrm{p}=0,000)$ de acordo com a classe social. As crianças classificadas nos níveis intermediário e alto apresentaram mais do que o dobro de chances para obesidade e sobrepeso (nível intermediário $\mathrm{RP}=2,33, \mathrm{IC}=1,77$ - 3,06 ; nível alto $\mathrm{RP}=2,64$, IC 1,93-3,62).

As variáveis referentes aos hábitos alimentares participaram somente da análise bivariada. As crianças que relataram tomar café da manhã tiveram 21,3\% menos chances de apresentar excesso de peso. O número de refeições diárias apresentou associação inversa com obesidade e sobrepeso. Os escolares que relataram fazer três refeições ou menos, por dia, apresentaram $48 \%$ mais chances para obesidade e sobrepeso.

\section{DISCUSSÃO}

As prevalências de obesidade (8\%) e sobrepeso (19,9\%) do presente estudo são semelhantes aos resultados encontrados nas cidades de Morro Reuter e Dois Irmãos - interior do RS, 7,5\% e 16,9\% de obesidade e sobrepeso respectivamente ${ }^{13}$. A pequena diferença de prevalências pode ser explicada pelos diferentes pontos de corte utilizados nos dois trabalhos.

Quando comparado a pesquisas de outras regiões do país, as prevalências de obesidade e sobrepeso encontradas são superiores aos estudos realizados em Fortaleza/CE ${ }^{10}$ (19,5\% de obesidade e sobrepeso), Corumbá/MS ${ }^{9}$ (6,5\% obesidade e 6,2\% sobrepeso) e Belo Horizonte/ $\mathrm{MG}^{18}$ (3,1\% obesidade e 8,4\% sobrepeso). Comparado com os resultados de Santos/SP12 (18\% obesidade e $15,7 \%$ sobrepeso), as prevalências de obesidade e sobrepeso do presente estudo são menores. Estas diferenças de prevalências com outros trabalhos realizados no país podem ser devido à diferença de faixas etárias estudadas ${ }^{10,18}$, diferentes pontos de corte utilizados ${ }^{9,12}$ e diferenças culturais de cada região.

Não foi encontrada diferença estatística significante entre as prevalências de obesidade e sobrepeso para meninos e meninas. Em Arapoti/ $\mathrm{PR}^{19}$, os investigadores também não encontraram diferença significativa em relação ao sexo. Dados divergentes foram encontrados na cidade de Presidente Prudente/SP, onde as prevalências de sobrepeso e obesidade foram maiores nos meninos ${ }^{20}$. Estas informações sugerem que o excesso de peso está distribuído diferentemente para meninos e meninas conforme a região avaliada. 
Tabela 1. Distribuição da amostra por idade, sexo, rede de ensino, IEN e prevalência de obesidade e sobrepeso.

\begin{tabular}{|c|c|c|c|c|c|}
\hline & & eutrófico & sobrepeso & obesidade & Intervalo de confiança* \\
\hline \multicolumn{6}{|l|}{ Idade } \\
\hline \multirow[t]{2}{*}{7 anos } & $\mathrm{n}$ & 110 & 28 & 11 & \\
\hline & $\%$ & $73,8 \%$ & $18,8 \%$ & $7,4 \%$ & $2,73-12,06$ \\
\hline \multirow[t]{2}{*}{8 anos } & $\mathrm{n}$ & 187 & 52 & 22 & \\
\hline & $\%$ & $71,6 \%$ & $19,9 \%$ & $8,4 \%$ & $5,92-10,87$ \\
\hline \multirow[t]{2}{*}{9 anos } & $\mathrm{n}$ & 207 & 49 & 20 & \\
\hline & $\%$ & $75,0 \%$ & $17,8 \%$ & $7,2 \%$ & $4,66-9,73$ \\
\hline \multirow[t]{2}{*}{10 anos } & $\mathrm{n}$ & 211 & 55 & 29 & \\
\hline & $\%$ & $71,5 \%$ & $18,6 \%$ & $9,8 \%$ & $7,79-11,80$ \\
\hline \multirow[t]{2}{*}{11 anos } & $\mathrm{n}$ & 189 & 63 & 22 & \\
\hline & $\%$ & $69,0 \%$ & $23,0 \%$ & $8,0 \%$ & $5,58-10,41$ \\
\hline \multirow[t]{2}{*}{12 anos } & $\mathrm{n}$ & 135 & 40 & 12 & \\
\hline & $\%$ & $72,2 \%$ & $21,4 \%$ & $6,4 \%$ & $2,40-10,39$ \\
\hline \multicolumn{6}{|l|}{ Sexo } \\
\hline \multirow[t]{2}{*}{ Meninas } & $\mathrm{n}$ & 521 & 147 & 53 & \\
\hline & $\%$ & $72,3 \%$ & $20,4 \%$ & $7,4 \%$ & $6,43-8,36$ \\
\hline \multirow[t]{2}{*}{ Meninos } & $\mathrm{n}$ & 518 & 140 & 63 & \\
\hline & $\%$ & $71,8 \%$ & $19,4 \%$ & $8,7 \%$ & $7,82-9,57$ \\
\hline \multicolumn{6}{|l|}{ Rede de Ensino } \\
\hline \multirow[t]{2}{*}{ Privada } & $\mathrm{n}$ & 143 & 42 & 14 & \\
\hline & $\%$ & $71,9 \%$ & $21,1 \%$ & $7,0 \%$ & $3,42-10,57$ \\
\hline \multirow[t]{2}{*}{ Pública } & $\mathrm{n}$ & 896 & 245 & 102 & \\
\hline & $\%$ & $72,1 \%$ & $19,7 \%$ & $8,2 \%$ & $7,45-8,87$ \\
\hline \multicolumn{6}{|l|}{ IEN } \\
\hline \multirow[t]{2}{*}{ Nível Baixo } & $\mathrm{n}$ & 103 & 19 & 3 & \\
\hline & $\%$ & $82,4 \%$ & $15,2 \%$ & $2,4 \%$ & $-7,59-12,39$ \\
\hline \multirow[t]{2}{*}{ Nível Intermediário } & $\mathrm{n}$ & 256 & 68 & 32 & \\
\hline & $\%$ & $71,9 \%$ & $19,1 \%$ & $9,0 \%$ & $7,24-10,75$ \\
\hline \multirow[t]{2}{*}{ Nível Alto } & $\mathrm{n}$ & 482 & 151 & 58 & \\
\hline & $\%$ & $69,8 \%$ & $21,9 \%$ & $8,4 \%$ & $7,46-9,33$ \\
\hline \multirow[t]{2}{*}{ TOTAL } & $\mathrm{n}$ & 1039 & 287 & 116 & \\
\hline & $\%$ & $72, .1 \%$ & $19,9 \%$ & $8,0 \%$ & $7,54-8,45$ \\
\hline
\end{tabular}

* sem a presença de sobrepeso e obesidade; ** Intervalo de confiança para prevalência de obesidade.

O presente estudo, diferentemente de outras pesquisas nacionais ${ }^{10,12,21}$, não encontrou diferença estatística significante entre excesso de peso em escolas públicas ou particulares $(p=0,32)$. Este achado pode ser explicado pelo nível sócioeconômico da amostra estudada, na qual 59\% dos escolares encontravam-se na classe mais alta do IEN, fato que poderia não diferenciar os escolares de escolas públicas e privadas. Também pode-se destacar que
$37 \%$ das crianças de classe alta (da amostra avaliada) estudam em escolas públicas.

Quando confrontadas as variáveis excesso de peso e nível sócioeconômico, os escolares com melhores condições econômicas apresentaram mais do que o dobro de chances para obesidade e sobrepeso. Estes achados são corroborados por outros estudos ${ }^{11,18}$, mostrando que o nível socioeconômico alto é fator de risco para obesidade e sobrepeso. 
Tabela 2. Características antropométricas e distância percorrida no teste de aptidão aeróbica.

\begin{tabular}{|c|c|c|c|c|c|}
\hline Idade & Sexo & Peso (kg) & Altura (m) & IMC $\left(\mathrm{kg} / \mathrm{m}^{2}\right)$ & Teste de $9 \mathrm{~min}^{*}(\mathrm{~m})$ \\
\hline \multirow[t]{2}{*}{7} & $\mathrm{~F}$ & $26,1 \pm 5,5$ & $1,24 \pm 0,57$ & $16,84 \pm 2,77$ & $1034 \pm 177$ \\
\hline & M & $26 \pm 4,3$ & $1,24 \pm 0,57$ & $17,03 \pm 2,19$ & $1028 \pm 206$ \\
\hline \multirow[t]{2}{*}{8} & $\mathrm{~F}$ & $28,5 \pm 4,9$ & $1,28 \pm 0,54$ & $17,38 \pm 2,46$ & $1045 \pm 160$ \\
\hline & M & $30,1 \pm 7,0$ & $1,29 \pm 0,60$ & $17,81 \pm 2,99$ & $1126 \pm 205$ \\
\hline \multirow[t]{2}{*}{9} & $\mathrm{~F}$ & $31,9 \pm 8,3$ & $1,33 \pm 0,66$ & $17,78 \pm 3,44$ & $1066 \pm 205$ \\
\hline & M & $32,2 \pm 6,7$ & $1,33 \pm 0,55$ & $17,89 \pm 3,18$ & $1157 \pm 182$ \\
\hline \multirow[t]{2}{*}{10} & $\mathrm{~F}$ & $36,6 \pm 9,6$ & $1,38 \pm 0,79$ & $18,84 \pm 3,55$ & $1086 \pm 163$ \\
\hline & M & $36,4 \pm 8,6$ & $1,39 \pm 0,64$ & $18,63 \pm 3,43$ & $1175 \pm 220$ \\
\hline \multirow[t]{2}{*}{11} & $\mathrm{~F}$ & $40,5 \pm 10,3$ & $1,44 \pm 0,76$ & $19,14 \pm 3,59$ & $1144 \pm 85$ \\
\hline & M & $40,5 \pm 9,8$ & $1,43 \pm 0,73$ & $19,54 \pm 3,77$ & $1195 \pm 231$ \\
\hline \multirow[t]{2}{*}{12} & $\mathrm{~F}$ & $45,01 \pm 1,2$ & $1,51 \pm 0,81$ & $19,43 \pm 3,86$ & $1146 \pm 191$ \\
\hline & M & $44,11 \pm 1,6$ & $1,48 \pm 0,81$ & $19,76 \pm 4,06$ & $1301 \pm 239$ \\
\hline \multirow[t]{2}{*}{ Total } & $\mathrm{F}$ & $35,2 \pm 10,6$ & $1,37 \pm 0,91$ & $18,33 \pm 3,44$ & $1090 \pm 185$ \\
\hline & M & $35,0 \pm 9,9$ & $1,36 \pm 0,97$ & $18,46 \pm 3,45$ & $1167 \pm 223$ \\
\hline
\end{tabular}

IMC= índice de massa corporal; DP= desvio padrão. * distância percorrida.

Tabela 3. Análise Bivariada e Multivariada entre Sobrepeso/Obesidade e variáveis do modelo teórico.

\begin{tabular}{|c|c|c|c|c|}
\hline & \multicolumn{2}{|c|}{ Análise Bivariada } & \multicolumn{2}{|c|}{ Análise Multivariada } \\
\hline & RP Bruta & Intervalo de Confiança & RP Ajustada & Intervalo de Confiança \\
\hline \multicolumn{5}{|c|}{ Indicador Econômico Nacional (1º nível) } \\
\hline Nível Baixo & 1,00 & & 1,00 & \\
\hline Nível Intermediário & 2,30 & $1,96-2,71$ & 2,33 & $1,77-3,06$ \\
\hline Nível Alto & 2,56 & $2,03-3,22$ & 2,64 & $1,93-3,62$ \\
\hline \multicolumn{5}{|l|}{ Tomar Café da Manhã } \\
\hline Não & 1,00 & & & \\
\hline Sim & 0,78 & $0,65-0,94$ & & \\
\hline \multicolumn{5}{|l|}{ Refeições diárias } \\
\hline 4 ou mais & 1,00 & & & \\
\hline Menos de 4 & 1,48 & $1,20-1,82$ & & \\
\hline \multicolumn{5}{|c|}{ Esportes fora do horário escolar ( $2^{\circ}$ nível) } \\
\hline Pratica & 1,00 & & 1,00 & \\
\hline Não pratica & 2,55 & $2,20-2,97$ & 1,41 & $1,11-1,81$ \\
\hline \multicolumn{5}{|c|}{ Hábitos Sedentários (TV, vídeo game, comp.) (3º nível) } \\
\hline Até 3 horas por dia & 1,00 & & 1,00 & \\
\hline Mais de 3 horas por dia & 3,07 & $2,46-3,83$ & 1,50 & $1,13-2,00$ \\
\hline \multicolumn{5}{|l|}{ Aptidão Aeróbica (4º nível) } \\
\hline Abaixo da zona saudável & 1,00 & & 1,00 & \\
\hline Dentro da zona saudável & 0,86 & $0,77-0,95$ & 0,59 & $0,46-0,77$ \\
\hline
\end{tabular}

$\mathrm{RP}=$ razão de prevalência.

Foi encontrada forte associação estatística entre excesso de peso e hábitos sedentários ( $\mathrm{p}=0,004$ e RP ajustada $=1,50$ ). Resultados semelhantes foram encontrados por Baruki et al. ${ }^{9}$, nos quais as crianças acima do peso gastavam mais tempo com atividades como leitura, vídeo game e televisão. Stettler, Signer e Suter ${ }^{22}$, encontraram risco maior para obesidade e sobrepeso em crianças que passavam 
mais tempo com atividades como jogos eletrônicos $(\mathrm{OR}=2,03)$ e assistir televisão $(\mathrm{OR}=2,83)$.

Com relação à prática de esportes fora do horário escolar, foi detectado $41,8 \%$ mais chances para obesidade e sobrepeso nas crianças que relataram não praticar esportes fora do horário escolar. Resultados semelhantes foram encontrados em Capão da Canoa/RS ${ }^{21}$ e Corumbá/MS ${ }^{9}$ onde as crianças consideradas menos ativas foram as que apresentaram maiores prevalências de obesidade e sobrepeso.

Os escolares que atingiram ou ultrapassaram a distância estipulada pelo teste de nove minutos apresentaram menores chances para excesso de peso (RP ajustada=0,59). Nassis, Psarra e Sidossis ${ }^{14}$, encontraram risco menor para obesidade em crianças com melhores condições cardiorrespiratórias. Crianças com melhores condições físicas tendem a apresentar um menor percentual de gordura corporal e uma menor prevalência de obesidade ${ }^{23}$.

A análise bivariada mostrou que as crianças que tomam café da manhã tiveram 21,3\% menos chances de apresentar excesso de peso. Triches e Giugliani ${ }^{13}$ encontraram resultados similares, ou seja, entre as crianças que tomavam café da manhã, $6 \%$ eram obesas e entre as que não tomavam $, 17,8 \%$ eram obesas $(\mathrm{OR}=3,4)$.

No presente estudo, o número de refeições diárias apresentou associação inversa com obesidade e sobrepeso. Fazer três refeições ou menos por dia aumentou em $48 \%$ as chances para obesidade e sobrepeso. Neutzling, Taddei e Gigante ${ }^{7}$ encontraram associação inversa entre excesso de peso e número de refeições diárias. Os escolares que faziam mais de três refeições diárias apresentaram risco menor para obesidade $(\mathrm{OR}=0,54)^{7}$. $\mathrm{O}$ fato da variável dos hábitos alimentares não permanecer no modelo sugere uma forte colinearidade com as demais variáveis estudadas, sugerindo que ambas estivessem medindo o mesmo parâmetro.

Como limitações do estudo, pode-se apontar o fato de ser um estudo transversal e como tal, não pode estabelecer causalidade, embora a força de algumas das associações encontradas possa sugerir isso. Além disso, poderia ter ocorrido um viés de memória em algumas questões como informações sobre a alimentação e número de horas gastas com atividades sedentárias. Apesar disso, a possibilidade das crianças subestimarem informações sobre os hábitos sedentários, por saber serem incorretos, reforçam os achados.

\section{CONCLUSÃO}

Em função de ter sido estudada amostra representativa da população alvo e terem ocorrido poucas perdas, pode-se dizer que os dados obtidos podem ser extrapolados à população alvo na faixa etária estudada.

O presente estudo mostrou que aptidão aeróbica e a prática de esportes fora do horário escolar são fatores que atuam positivamente para manutenção de um peso adequado. Já a manutenção de hábitos sedentários, não tomar café da manhã e realizar um número inferior a quatro refeições diárias favorecem, nas crianças, peso acima do normal. As crianças pertencentes às classes socioeconômicas mais altas, apresentaram prevalências de excesso de peso mais elevadas.

Considerando as limitações do estudo, pode-se dizer que as prevalências de obesidade e sobrepeso da população estudada encontram-se elevadas e devem ser motivo de preocupação dos sistemas de saúde do município e da sociedade.

\section{REFERÊNCIAS BIBLIOGRÁFICAS}

1. Wang Y, Monteiro C, Popkin BM. Trends of obesity and underweight in older children and adolescentes in the United States, Brazil, China and Rússia. Am J Clin Nutr 2002;75(6):971-977.

2. Taylor ED, Theim KR, Mirch MC, Ghorbani S, Tanofsky-kraff M, Adler-Wailes DC, et al. Orthopedic complications of overweight in children and adolescents. Pediatrics 2006;117(6):2167-2174.

3. US Preventive Service Task Force. Screening and interventions for overweight inchildren and adolescents: recommendation statement. Pediatrics 2005;116(1):205-209.

4. Hughes AR, Farewell K, Harris D, Reilly JJ. Quality of life in a clinical sample of obese children. Int J Obes 2007;31(1):39-44.

5. Deshmukh-Taskar P, Nicklas TA, Morales M, Yang S-J, Zakieri I, Berenson GS. Tracking of overweight status from childhood to young adulthood: the Bogalusa Heart Study. Eur J Clin Nutr 2006; 60(1):48-57.

6. Freedman DS, Mei Z, Srinivasan SR, Berenson GS, Dietz WH. Cardiovascular risk factors and excess adiposity among overweight children and adolescents: The Bogalusa Heart Study. J Pediatr 2007;150(1):12-17.

7. Neutzling MB, Taddei JAAC, Gigante DP. Risk factors of obesity among Brazilian adolescentes: a case-control study. Public Health Nutr 2003;6(8):743-749.

8. Batista Filho M, Rissin A. A transição nutricional no Brasil: tendências regionais e temporais. Cad Saúde Pública 2003;19(supl.1):S181-S191.

9. Baruki SBS, Rosado LEFPL, Rosado GP, Ribeiro RCL. Associação entre estado nutricional e atividade física em escolares da Rede Municipal de Ensino em Corumbá - MS. Rev Bras Med Esporte 2006;12(2):90-94.

10. Campos LA, Leite AJM, Almeida PC. Prevalência de sobrepeso e obesidade em adolescentes escolares do município de Fortaleza, Brasil. Rev Bras Saúde Matern Infant 2007;7(2):183-190. 
11. Celi F, Bini V, De Giorgi G, Molinari D, Faraoni F, Di Stefano G, et al. Epidemiolgy of overweight and obesity among school children and adolescentes in three provinces of central Italy, 1993-2001: study of potential influencing variables. Eur J Clin Nutr 2003;57(9):1045-1051.

12. Costa RF, Cintra IP, Fisberg M. Prevalência de sobrepeso e obesidade em escolares da cidade de Santos - SP. Arq Bras Endocrinol Metab 2006;50(1):60-67.

13. Triches MR, Giugliani ERJ. Obesidade, práticas alimentares e conhecimentos de nutrição em escolares. Rev Saúde Pública 2005;39(4):541-547.

14. Nassis GP, Psarra G, Sidossis LS. Central and total adiposity are lower in overweight and obese children with high cardiorespiratory fitness. Eur J Clin Nutr 2005;59(1):137-141.

15. Barros AJD, Victora CG. Indicador econômico para o Brasil baseado no censo demográfico de 2000. Rev Saúde Pública 2005;39(4):523-529.

16. Setor de Pedagogia do Esporte do CENESP-UFRGS. PROESP-BR, Projeto Esporte Brasil - Indicadores de Saúde e Fatores de Prestação Esportiva em Crianças e Jovens. Manual de Aplicação de Medidas e Testes Somatomotores. Perfil 2002;6(6):9-34.

17. Cole TJ, Bellizzi MG, Flegal KM, Dietz WH. Establishing a standard definition for child overweight and obesity worldwide: international survey. BMJ 2000;320(6):1240-1245.

18. Ribeiro RQC, Lotufo PA, Lamounier JA, Oliveira GO, Soares JF, Botter DA. Fatores adicionais de risco cardiovascular associados ao excesso de peso em crianças e adolescentes: o estudo do coração de Belo Horizonte. Arq Bras Cardiol 2006;86(6):408-418.
19. Salomons E, Rech CR, Lock MR. Estado nutricional de escolares de seis a dez anos de idade da rede municipal de ensino de Arapoti, Paraná. Rev Bras Cineantropom Desempenho Hum 2007;9(3):244-249.

20. Fernandes RA, Kawaguti SS, Agostini L, Oliveira AR, Ronque ERV, Freitas Junior IF. Prevalência de sobrepeso e obesidade em alunos de escolas privadas do município de Presidente Prudente - SP. Rev Bras Cineantropom Desempenho Hum 2007;9(1):21-27.

21. Suñé FR, Dias-da-Costa JS, Olinto MTA, Pattussi MP. Prevalência e fatores associados para sobrepeso e obesidade em escolares de uma cidade no Sul do Brasil. Cad Saúde Pública 2007;23(6):1361-1371.

22. Stettler N, Signer TM, Sutter PM. Eletronic games and environmental factors associated with childhood obesity in Switzerland. Obes Res 2004;12(6):896-903.

23. ACSM. Diretrizes do ACSM para os testes de esforço e sua prescrição. 7. ed. Rio de Janeiro: Guanabara Koogan; 2007.

\footnotetext{
Endereço para correspondência

Ricardo Rodrigo Rech

Francisco Getúlio Vargas, 1130 - Vila Olímpica

- Bloco 70. Curso de Educação Física.

CEP 95070-560 - Caxias do Sul - RS

E-mail: ricardo.rech@gmail.com
} 